\title{
Identification of community-consensus clinically relevant variants and development of single molecule molecular inversion probes using the $\mathrm{CIViC}$ database
}

Erica K. Barnell ${ }^{1,2}$, Adam Waalkes ${ }^{3}$, Kelsi Penewit ${ }^{3}$, Katie M. Campbell ${ }^{1,4}$, Zachary L. Skidmore ${ }^{1}$, Colin C. Pritchard ${ }^{3}$, Todd A. Fehniger ${ }^{5,6}$, Ravindra Uppaluri ${ }^{7}$, Ramaswamy Govindan ${ }^{2,5}$, Malachi Griffith ${ }^{1,2,5,6}$, Stephen J. Salipante ${ }^{3+}$, Obi L. Griffith ${ }^{1,2,5,6+}$

${ }^{1}$ McDonnell Genome Institute, Washington University School of Medicine, St. Louis, MO, USA

${ }^{2}$ Department of Genetics, Washington University School of Medicine, St. Louis, MO, USA;

${ }^{3}$ Department of Laboratory Medicine, University of Washington, Seattle, WA, USA

${ }^{4}$ Department of Medicine, Division of Hematology-Oncology, University of California, Los Angeles, CA, USA.

${ }^{5}$ Siteman Cancer Center, Washington University School of Medicine, St. Louis, MO, USA.

${ }^{6}$ Division of Oncology, Department of Medicine, Washington University School of Medicine, St. Louis, MO, USA.

${ }^{7}$ Department of Surgery/Otolaryngology, Brigham and Women's Hospital and Dana-Farber Cancer Institute, Boston, MA, USA.

+Corresponding author

Keywords: Precision oncology; single-molecule molecular inversion probe; Clinical Interpretations of Variants in Cancer database; capture panel

\section{Abstract}

Clinical targeted sequencing panels are important for identifying actionable variants for cancer patients, however, there are currently no strategies to create impartial and rationally-designed panels to accommodate rapidly growing knowledge within the field. Here we use the Clinical Interpretations of Variants in Cancer database ( $\mathrm{CIViC}$ ) in conjunction with single-molecule molecular inversion probe (smMIP) capture to identify and design probes targeting clinically relevant variants in cancer. In total, 2,027 smMIPs were designed to target 111 eligible CIViC variants. The total genomic region covered by the CIViC smMIPs reagent was $61.5 \mathrm{~kb}$. When compared to existing genome or exome sequencing results ( $n=27$ cancer samples from 5 tumor types), CIViC smMIP sequencing demonstrated a $95 \%$ sensitivity for variant detection ( $n=61 / 64$ variants). Variant allele frequency for variants identified on both sequencing platforms were highly concordant (Pearson correlation $=0.885$; $n=61$ variants). Moreover, for individuals with paired tumor/normal samples ( $n=12), 182$ clinically relevant variants missed by original sequencing were discovered by CIViC smMIPs sequencing. This design paradigm demonstrates the utility of an open-sourced database built on attendant community contributions for each variant with peer-reviewed interpretations. Use of a public repository for variant identification, probe development, and variant annotation could provide a transparent approach to build a dynamic next-generation sequencing-based oncology panel. 


\section{Introduction}

Despite recognition that genomics plays an important role in tumor prognosis, diagnosis, and treatment, scaling genetic analysis to encompass every clinical tumor specimen has been unattainable. ${ }^{1,2}$ Barriers preventing widespread adoption of genomic analysis into treatment protocols include: costs associated with genomic sequencing and analysis, ${ }^{3}$ computational limitations preventing timely and accurate annotation of variants, ${ }^{3}$ and extensive growth of knowledge within the precision oncology field. ${ }^{4}$ Technological improvements in sequencing and data analysis continue to reduce these first two limitations, however, less progress has been made in integrating dynamic genomic annotation into clinical diagnoses. Over $22 \%$ of oncologists have acknowledged limited confidence in their own understanding of how genomic knowledge applies to patients' treatment and $18 \%$ reported testing patients' genetics infrequently. ${ }^{5}$ In the face of exponential growth in clinically relevant genomic findings driven by precision oncology efforts, there will likely be increased inability for physicians to command the most current information, resulting in increasing delay between academic discovery and clinical integration of variant information. This information gap between the knowledge of variant function and its clinical integration has been described as the "interpretation bottleneck". ${ }^{4-6}$

Alleviating the interpretation bottleneck will require co-development of targeted sequencing panels and bioinformatic tools that effectively elucidate and annotate clinically actionable variants from sequencing data. ${ }^{7,8}$ These two requirements each raise separate challenges. Commercial and academic pan-cancer clinical gene capture panels have now become commonplace, with at least two obtaining Food and Drug Administration (FDA) approval (FoundationONE CDx ${ }^{9}$ and MSK-IMPACT ${ }^{10}$ ). Even so, few panels indicate how genomic loci are selected for panel inclusion (Supplementary Table 1), and none have proposed a sustainable or scalable mechanism to allow for panel evolution over time in response to knowledge advances in molecular oncology. Variant interpretation perhaps poses even greater and more persistent challenges. Commercial strategies typically rely on the manual curation and organization of research findings into structured databases, which are expensive to create and maintain, forcing companies to limit public access or to charge for use. The resulting lack of transparency creates inefficiencies in the field through unnecessary replication of experiments and suboptimal communication with clinicians, ultimately hindering development of effective patient treatment plans. Separately, governmental and academic institutions have developed variant interpretation resources like COSMIC ${ }^{11}$, ClinVar ${ }^{12}$, and $\mathrm{CBioPortal}^{13,14}$ that have drastically improved research efforts and academic discovery, however, these resources do not have well-described clinical relevance summaries for all variants that can be easily accessed and utilized by physicians. Many resources that do provide detailed clinical interpretation of cancer variants (e.g., oncoKB ${ }^{15}$, Cancer Genome Interpreter ${ }^{16}$, Clinical Knowledgebase ${ }^{17}$, and others) but these databases are either limited by license restrictions or closed curation models. The OncoPaD ${ }^{18}$ portal provided the first prospective method to create a rational design for gene panels by linking clinically relevant variants to genomic loci based on a cohort of tumor samples, but it is not linked to detailed and actively updated clinical interpretations.

To address these limitations, here we describe a strategy to integrate a publicly sourced, open variant knowledgebase with an inexpensive and modular platform for targeted gene enrichment and 
error-corrected sequencing. The Clinical Interpretations of Variants in Cancer (CIViC) database is a freely-accessible, publicly curated repository of therapeutic, prognostic, predisposing, and diagnostic information in precision oncology that permits rapid integration of academic information into an accessible platform for scientists, physicians, and patients ${ }^{19}$ (Supplementary Figure 1). As a proof-of-principle, we utilized this knowledgebase to inform rational design of a targeted sequencing reagent for pan-cancer relevant genetic variants through single-molecule molecular inversion probe (smMIP) capture, which integrates multiplexed targeted sequencing with unique molecular identifier (UMID)-mediated sequence error correction. smMIP capture provides an inexpensive, scalable, and modular platform for targeted gene sequencing, which can be scaled to ultrasensitive levels of variant detection, and which can accommodate the integration of additional probes in response to evolving assay needs. Combined, these two technologies provide a testing framework that links physical genomic loci to relevant clinical information. In this study, we first assess the feasibility of using an open-sourced database to impartially identify clinically relevant variants and design smMIPs probes for their identification. The reagent design was evaluated by comparing CIViC smMIP capture results to orthogonal exome sequencing for 27 cancer samples (Figure 1). Ultimately, we hope that this research will inform development of a clinically validated panel that can dynamically integrate new evidence in precision oncology, enabling systemization of methods for detecting and interpreting cancer variants.

\section{Materials and Methods}

\section{CIViC actionability score development}

A CIViC actionability score was created for each variant within the CIViC database to establish whether the existing level of curated evidence warrants inclusion for smMIP targeted capture. The CIViC actionability score determines: 1 ) the strength of the evidence that was curated and 2) the total level of curation that has been completed for each variant. To determine evidence strength, the evidence level score and the trust rating score were calculated. The evidence level score is a 10-point scale that weighs the evidence strength based on category. Broadly, highest points are awarded to large clinical studies and lower points are awarded to case studies, in vitro studies, and inferential evidence. The trust rating score is a 5-star scale that reflects the curators confidence in the quality of the study within those categories. To determine the total level of curation for each variant, evidence level scores were multiplied by trust rating scores and summed across all evidence items (Supplementary Figure 2). This final value (i.e., the CIViC actionability score) was implemented into the CIViC database using the Variant Application Programming Interface (API) endpoint.

\section{Determining eligible CIViC variants for smMIP capture}

Variants that attained a CIViC actionability score of greater than 20 points were eligible for smMIP targeting. After filtering on the CIViC actionability score, variants were filtered using the curated Sequence Ontology IDs (SOIDs) to only include variants that can be analyzed using a DNA-based sequencing platform. Within CIViC, SOIDs were manually grouped as either: "DNA-based", "RNA-based", and/or "Protein-based" (Supplementary Table 2). For example, variants with the Variant Type of "missense_variant" would be labeled as "DNA-based," whereas variants with the 
Variant Type of "transcript_variant" would be labeled as "RNA-based". Variants that had a "DNA-based" SOID were eligible for the smMIP targeting and variants whose SOIDs were "RNA-based" and/or "Protein-based" were ineligible. Eligible variants were further filtered if any of the following applied: 1) all evidence supported only germline clinical relevance, 2) evidence was directly conflicting (e.g., one evidence statement detailed sensitivity to a therapeutic and a different evidence statement detailed resistance to the same therapeutic in the same disease setting), or 3) a majority of evidence in a bucket variant (e.g., MUTATION) pointed to a hotspot that was already being covered.

\section{Designing smMIPs for the CIViC capture reagents}

All eligible variants were further categorized by variant length. Using $\mathrm{CIViC}$ curated coordinates, variant length was determined (i.e., variant start position minus variant stop position), to determine the number of smMIPs probes required to adequately assess each variant. If the variant length was $<250$ base pairs, the variant was eligible for hotspot targeting. If the variant was $>250$ base pairs, the variant required tiling of the protein coding exons. Some large-scale copy number variants (i.e., "AMPLIFICATION", "LOSS", "DELETION"), were eligible for sparse tiling, wherein 10 probes distributed across the exons of the gene were retained to enable assessment of copy number state. Other variant types such as "MUTATION", or "FRAMESHIFT MUTATION", etc., required tiling of all protein coding exons (Supplementary Table 3).

For variants that required hotspot targeting, smMIPs probes were designed for the genomic region indicated in the $\mathrm{CIViC}$ database. For all variants that required sparse exon tiling or full exon tiling, the representative transcript from the $\mathrm{CIViC}$ database was used to obtain all possible exons associated with each gene from Ensembl (biomart="ENSEMBL_MART_ENSEMBL", host="grch37.ensembl.org", dataset="hsapiens_gene_ensembl"). Exons were filtered by Biotype to remove untranslated regions. For variants that required sparse exon tiling, approximately 10 smMIPs were designed to cover a portion of the transcript. For variants that required full exon tiling, overlapping smMIPs (i.e., at least one basepair of overlap) were designed to tile across all protein coding exons in the gene that encompassed the variant. smMIPs were designed and synthesized as previously described with the single alteration that the "-double_tile_strands_separately" flag was used to separately capture each strand of DNA surrounding the target. ${ }^{20}$

\section{smMIP sequencing and data analytics}

Sequencing library construction and balancing of the probe pool were performed as described previously ${ }^{20}$, and sequencing was performed using an Illumina Nextseq 500. Probes were excluded from the final reagent if they demonstrated poor hybridization to target sequence during initial quality checks.

Sequence data analysis was performed as previously described ${ }^{20}$ with three enhancements. First, consensus reads were generated using fgbiotools (http://fulcrumgenomics.github.io/fgbio/) CallMolecularConsensusReads utility with parameters "--error-rate-post-umi=30 --min-reads=2 --min-input-base-quality 20 ". Second, a custom variant caller was utilized to identify all consensus calls at a site having at least 2 supporting reads with a minimum specified mapping quality (mapping quality score $>0$ ). Third, variants were required to be detected on at least four DNA strands (at least 2 positive and at least 2 negative) in order to be considered real, rather than post-biological artifact. ${ }^{21}$ 
Collectively, these provisions require that at least two reads are derived from a common UMID to create a consensus read and that multiple consensus reads in both directions support the apparent variant. This helps to exclude pre-analytic artifacts reflecting DNA damage and stochastic errors that occur during library construction and sequencing.

\section{Orthogonal sequencing and data analytics}

Orthogonal sequencing data from previously conducted whole exome or genome sequencing was used to validate variants identified using CIViC smMIP sequencing. Sequencing alignment and somatic variant calling for the AML31 sample was performed according to Griffith et al. ${ }^{22}$ Briefly, reads were aligned to GRCh37 using BWA v0.5.923 and variants were called using one of seven variant callers listed in the manuscript. Sequencing data from the SCLC cases, OSCC cases, and HL cases were analyzed using the Genome Modeling System ${ }^{2}$ at the McDonnell Genome Institute. Reads from these studies were aligned to the reference genome (hg19/GRCh37 or hg38/GRCh38) using BWA-MEM v0.7.10 $0^{24}$ and duplicates were marked by Picard ${ }^{25}$ and/or SAMBLASTER v0.1.22. ${ }^{26}$. For the SCLC cases, Single nucleotide variants (SNVs) were called using SomaticSniper ${ }^{27,28}$, VarScan $^{29}$, and Strelka ${ }^{30}$; small insertions and deletions (indels) were called using GATK ${ }^{31}$, Pindel ${ }^{32}$, VarScan2 ${ }^{33}$, and Strelka. For OSCC cases, SNVs were detected using SomaticSniper v1.0.4, VarScan2 v2.3.6, Strelka v1.0.11, SAMtools $r 982^{34}$, and Mutect v1.1.4 $4^{35}$. Small indels were detected by GATK v5336 $6^{36}$, VarScan2, Strelka, and Mutect. For HL cases, SNVs were called using the intersection of SomaticSniper v1.0.4, VarScan v2.3.6, Strelka v1.0.11, and Mutect v1.1.4, and indels were called using GATK, Pindel v0.5, VarScan v2.3.6, and Strelka v1.0.11. For these three cohorts, variants identified by automated callers were subjected to heuristic filtering (removal of variants with low VAF $[<5 \%$ ] or low coverage [ $<20 \mathrm{X}$ in tumor or normal track]) and false positives were removed via manual somatic variant refinement. ${ }^{37}$ If coordinates were aligned to $\mathrm{GRCh} 38$, final variants were lifted over to GRCh37 using LiftOver ${ }^{38}$ For the CRC cohort, sequencing, variant calling, and clinical annotation were performed according to methods highlighted in Pritchard et al. ${ }^{39}$ Briefly, sequencing was performed using Illumina next-generation sequencing (Illumina, San Diego, CA) and sequencing reads were aligned using BWA v0.6.1-r104 and SAMtools v0.1.18. Indel realignment was then performed using GATK v1.6 and duplicate reads were removed using Picard v1.72. SNV and indel calling was performed through the GATK Universal Genotyper using default parameters and VarScan v2.3.2.

\section{Rescue and annotation of clinically relevant variants}

We compared the variants identified by the CIViC smMIP sequencing pipeline to the variants called by original exome or genome sequencing for samples that had matched tumor and normal sequencing. Variants that were specific to the CIViC smMIP sequencing pipeline were further evaluated for variant support in the original exome or genome sequencing. Using the manual review guidelines outlined in Barnell et. al. ${ }^{37}$, we manually reviewed genomic loci for smMIPs-only variants using both the smMIPs aligned BAM files and the original exome or genome aligned BAM files. Variants were grouped into four categories based on review of smMIP and original sequencing: 1) germline polymorphism, 2) pipeline artifact, 3) variant support on smMIP sequencing but no variant support on original sequencing, 4) variant support on both smMIP sequencing and original sequencing. smMIPs-only variants were considered germline polymorphisms if there was sufficient 
support for the variant in the normal track. Variants were considered attributable to pipeline artifacts if aligned reads showed low variant support and/or reads indicated poor mapping/alignment. The remaining variants, regardless of the extent of support in original sequencing, were annotated using curated coordinates in the CIViC database. smMIP-only variants derived from tumor-only samples were further filtered using gnomAD ${ }^{40}$ and annotated using the $\mathrm{CIViC}$ database. For variants that showed support on smMIPs sequencing but no variant support on original sequencing, we calculated the likelihood that the variant would be detected using the original coverage and the observed smMIPs VAF. Given that original sequencing required at least 4 reads for a variant to be called somatic, we calculated the binomial probability of obtaining $\leq 3$ variant-supporting reads given the original coverage (number of chances to get a variant supporting read) and the observed smMIPs variant allele frequency (likelihood that a read would show variant support). If the binomial probability of $\leq 3$ variant-supporting reads was $>95 \%$, then it was considered statistically unlikely that a variant would be called using original sequencing data.

\section{Code and accessibility}

All raw data, analysis, and preprocessing code, are publically available on the GitHub repository. All plots were produced using the MatPlotlib library in Python. ${ }^{41}$ The raw sequencing data are publically available for most projects included in this study (Supplementary Table 4). The smMIP sequence analysis pipeline is accessible on bitbucket.

\section{URLS}

Clinical Interpretation of Variants in Cancer database, http://www.civicdb.org/. CIViC Interface public API, http://griffithlab.org/civic-api-docs/. CIViC Panels API https://civicdb.org/api/panels. GitHub, https://github.com/. smMIPs GitHub Repo,

https://github.com/griffithlab/civic-panel/tree/master/smMIPs_Manuscript. Ensembl GRCH38 BioMart, http://useast.ensembl.org/biomart/martview/c2a89c09bd76058dfd5a9821453a1da7. smMIP sequence analysis pipeline, https://bitbucket.org/uwlabmed/smmips analysis.

\section{Results}

\section{Identification of eligible CIViC variants for smMIP targeting}

At the time of CIViC smMIPs reagent design, there were 988 variants spanning 275 genes within the CIViC database that had at least one evidence item. After filtering based on CIViC actionability score and SOID (see Methods), smMIPs were designed to cover all eligible CIViC variants. A set of 2,097 probes were developed and tested on control samples. Of these, 70 probes showed poor capture efficiency and were subsequently eliminated from the analysis. Removal of the underperforming probes affected 32 variants across 16 genes. The final capture reagent targeted $111 \mathrm{CIViC}$ variants spanning approximately $61.5 \mathrm{~kb}$ of genomic space (Supplementary Table 3 ). Of these variants targeted by smMIP capture, 71 required hotspot targeting, 14 variants required sparse exon tiling, and 26 required full exon tiling. The 111 variants covered by CIViC smMIP sequencing were based on 1,168 clinically relevant evidence items whereby 820 (70\%) evidence items predicted response to 
a therapeutic, $232(20 \%)$ detailed prognostic information, $52(4 \%)$ indicated diagnostic information, and $64(6 \%)$ evidence items supported predisposition to cancer (Figure 2).

After reagent development, the $\mathrm{CIViC}$ database was re-evaluated to determine additional variants that did not meet the original actionability score threshold, but were encapsulated by the designed smMIPs. The total number of variants covered by the CIViC smMIPs, regardless of actionability score, was 408 variants spanning 49 genes. The majority of these additional variants $(n=203)$ were contained within $\mathrm{VHL}$, an area of very active CIViC curation.

\section{Tumor samples used to validate CIViC smMIPs design}

We assembled validation samples from 5 different cancer genomic studies. Four studies were conducted at the McDonnell Genome Institute at Washington University School of Medicine and one study was performed at the University of Washington (Supplementary Table 4). In total, we obtained tumor and paired normal samples from 5 individuals with head and neck squamous cell carcinoma (HNSCC), 9 individuals with small cell lung cancer (SCLC) ${ }^{42}$, and 1 individual with Hodgkin's lymphoma $(\mathrm{HL})$. We also obtained tumor-only samples from 1 individual with $\mathrm{HL}, 1$ individual with acute myeloid leukemia (AML) ${ }^{22}$, and 5 individuals with colorectal cancer (CRC). In total, there were 37 samples evaluated from 22 individuals.

Each of the 22 individuals had previously undergone exome or genome sequencing, automated somatic variant calling, and somatic variant refinement via manual review (see Methods). Using exome or genome sequencing the total number of putative somatic variants called for these 22 samples was 12,602 . The average variant burden was 573 variants per sample with a range of 2 to 3,900 variants per sample. The variant coordinates from these samples were compared to the genomic region covered by the CIViC smMIP capture to determine potential validating variants. In total, there were 84 variants identified via exome or genome sequencing that overlapped with the CIViC smMIPs region assayed (Supplementary Table 5).

\section{smMIP sequencing and data analysis}

\section{Initial quality check}

The average number of total tags captured for all samples was 5.4 million (standard deviation $=3.3$ million tags). Two HNSCC tumor samples had significantly fewer reads than the rest (i.e., greater than 1 standard deviation from the mean) and one $\mathrm{HL}$ sample had reduced tag complexity relative to the rest (i.e., fewer than 600,000 unique captured mips). These results are consistent with poor template quality and/or quantity, and the samples were excluded from the analysis. There were seven normal samples that had either fewer reads or low complexity due to low input mass (100-200 ng). Post quality check, 33 samples derived from 19 individuals were eligible for reagent validation. These samples had 65 variants derived from orthogonal sequencing that had overlap with the CIViC smMIPs coverage (Figure 3). The average consensus read depth for these 65 variants was 2,942 reads (std $=4,697$ reads).

Accuracy of CIViC smMIP variant identification compared to exome or genome variant identification 
Of the 65 variants identified on exome sequencing, all but 4 were also identified using CIViC smMIP sequencing (Figure 3). One variant was missed due to lack of adequate coverage, two variants were missed due to low performing probes, and one variant was retrospectively considered ineligible due to smMIPs design. The variant missed due to inadequate coverage was a TP53 (p.G266R) variant on AML31. Original sequencing indicated that this variant was present at $0.04 \%$ VAF, therefore, given smMIPs coverage of 2,388 reads at this site, there was only a $0.01 \%$ chance that this variant would have been detected (one-tailed probability of exactly, or greater than, 4 reads $(\mathrm{K})$ out of 2,388 reads $(n) ; p=.004603)$. However, this low-prevalence variant could have been recovered given additional sequence coverage. Additionally, there were two variants missed due to low MIP performance. The first variant that was missed (PTEN - C71Y on SCLC8 at 94\% VAF) was due to poor performance of the MIP covering the region of interest in the reverse direction. This MIP showed only 1 aligned read across all 36 samples and had no aligned reads in SCLC8. Despite the fact that there was extensive support from the forward MIP (95\% VAF with 34 / 35 consensus reads), the requirement that both forward and reverse reads show support prevented this variant from being called (see Methods). The second missed variant (PTEN - e8-1 on SCLC4 at 100\% VAF) was due to low performance of MIPs in both directions. Even though both the forward and the reverse MIP showed variant support, the forward MIP only contained 2 consensus reads and the reverse MIP only contained 1 consensus read, preventing it from being called as somatic. The final variant (STK - T328N on CRC5 at 32\% VAF) was retrospectively considered ineligible because the original smMIPs developed to cover the eligible STK variant called for sparse tiling (i.e., identification of copy number change). As such, the variant was contained by a region that did not have full coverage in the forward direction. When evaluating the reverse MIP that contained this site, we observed a 34\% VAF (402 / 1,184 reads), which was comparable to original sequencing. However, lack of a secondary probe designed against the complementary DNA strand prevented this variant from being called as somatic. After removing this variant from the list of eligible variants, the CIVIC smMIP capture sequencing attained a $95 \%$ sensitivity for variant detection ( $n=64$ variants).

\section{VAF correlation between CIViC smMIPs sequencing and exome or genome sequencing}

Variant allele frequencies (VAF) obtained via exome or genome sequencing were compared to the VAF obtained using the CIViC smMIPs. To compare VAF quantitation across platforms, the 19 variants obtained from samples that failed CIViC smMIPs sequencing quality check were eliminated (Figure 4a). Subsequently, we eliminated the four variants that were not validated using the CIViC smMIPs reagents (Figure $\mathbf{4 b}$ ). When comparing original VAF to CIViC smMIPs VAFs, Pearson correlation for the remaining 61 variants was 0.885 . There were several variants whereby the VAF observed on the CIViC smMIPs sequencing was lower than that observed on the original sequencing. These outliers were not associated with tumor type, sequencing mass input, average coverage, presence of matched normal, or sample type (Figure $4 \mathbf{c - g}$ ).

\section{Analysis of variants only identified using CIViC smMIP sequencing}

\section{CIViC smMIPs capture rescues clinically relevant variants}

Using samples that had sequencing data for both tumor and matched normal ( $\mathrm{n}=12$ samples), we evaluated whether the targeted CIViC smMIP sequencing could identify clinically relevant variants 
that had been missed during the original sequencing annotation pipeline. There were 273 variants recovered by $\mathrm{CIViC}$ smMIP sequencing that were not identified using exome or genome sequencing. After manually reviewing these variants within the original exome or genome alignments, 55 variants (20.1\%) were identified as germline mutations. smMIP sequencing VAF distribution at $50 \%$ and $100 \%$ further supported that these variants were germline polymorphisms (Figure 5a). An additional 36 variants $(13.2 \%)$ were thought to be caused by pipeline artifacts and attributable to assumptions underlying automated callers or alignment problems. The majority of these artifacts were caused by nucleotide repeats in the reference sequence (Figure $\mathbf{5 b}$ ). There were $171(62.6 \%)$ variants called as somatic using CIViC smMIPs that did not have any variant support on the original sequencing. For these variants, we calculated the binomial probability that $\leq 3$ reads would support the variant given the original coverage (number of chances to get a variant supporting read) and the observed smMIPs variant allele frequency (likelihood that a read would show variant support). If the binomial probability of $\leq 3$ variant-supporting reads was $>95 \%$, then it was considered statistically unlikely that a variant would be called using original sequencing data. Using this calculation, 162 variants $(94.7 \%)$ showed insufficient coverage in the original exome or genome sequencing for detection (Figure 5c). Finally, 11 variants $(4.2 \%)$ were not called as somatic on original sequencing but did show some variant support in those original sequencing data. The VAFs observed on original sequencing data were strongly correlated with the VAFs observed using CIViC smMIP sequencingl (Pearson $r=0.92$ ) (Figure 5d). Reviewing manual review files from the original sequencing, we observed that 6 of these variants failed manual review due to low VAF, 4 variants had not been called by automated somatic variant callers, and 1 variant failed manual review due to a perceived sequencing artifact.

We elucidated the clinical relevance of the 182 variants that were missed by original sequencing but legitimately called as somatic by CIViC smMIP sequencing (i.e., not germline or caused by pipeline artifact). In total, there were $73 \mathrm{CIViC}$ evidence statements associated with variants in the 12 samples that had both tumor and matched normal sequencing data (Supplementary Table 6). Of these, 33 were predictive, 4 were diagnostic, 36 were prognostic, and 0 were predisposing.

\section{Annotation of CIViC smMIPs variants using tumor-only samples}

We also examined the clinical relevance of the 401 variants in tumor-only samples that were called as somatic by CIViC smMIP sequencing. In total, there were $130 \mathrm{CIViC}$ evidence statements associated with the 6 samples that had tumor-only sequencing data. For these $130 \mathrm{CIViC}$ evidence statements, 63 were predictive, 1 was diagnostic, 66 were prognostic, and 0 were predisposing.

\section{Discussion}

The method employed to build a preliminary $\mathrm{CIViC}$ smMIPs targeted sequencing reagent offers several advantages relative to existing design paradigms. Use of an open-sourced database provides an unbiased mechanism to survey existing literature within precision oncology to identify variants that are relevant for capture. Additionally, the public CIViC API permits rapid mapping of identified somatic and germline variants to $\mathrm{CIViC}$ clinical relevance summaries. Most importantly, the variants covered by $\mathrm{CIViC}$ and associated clinical summaries can be updated in real-time as knowledge is entered into the database to accommodate new information discovered within the field of precision oncology. The choice to use the smMIP capture method for sequencing provides inherent error correction capability, 
scalability to detect ultrasensitive variation, and cost effectiveness within a modular design.

Combining the public access CIViC database with an ultrasensitive and versatile capture reagent thus provides an advantageous and principled method for building precision oncology capture reagents. This approach could enable a standardized framework for detecting and interpreting cancer-relevant genomic variation, lowering barriers to use of genomic analysis in the clinical practice of oncology.

The CIViC actionability score was used to obtain a community consensus on the clinical relevance for all variants within the database and the publically curated coordinates were used to design the probes required for variant capture. By comparing variants identified using exome or genome sequencing with variants identified using CIViC smMIPs sequencing, we concluded that CIViC variants and variant coordinates were being accurately incorporated into the database. Overall, the design attained an accuracy of $95 \%$ ( $n=64$ variants) with Pearson $r$ correlation of 0.885 for VAFs of variants identified by both sequencing methods. This finding validates the technological approach of the smMIPs design and that coordinates derived from the CIViC knowledgebase can be used to accurately cover desired variants of interest.

This study used the CIViC actionability score to determine if a variant had sufficient evidence to influence clinical decisions. Filtering out variants with an actionability score $<20$ points ensured that smMIPs were designed to only target loci that had clinical implications if mutated. In addition to defining relative level of variant curation, the CIViC actionability score can also be used identify gaps within the database. For example, if an externally validated variant (e.g., IDH1 - R132S) has a low score, users can search the queue of suggested publications in CIViC or find an article to add an evidence statement. Additional curation would subsequently increase the actionability score to better reflect the clinical value of the variant. Addition of the CIViC actionability score to the interface should promote efficient use of curation resources, filling knowledge gaps, and helping to understand when variants have achieved adequate curation coverage.

There were only three orthogonally validated variants that were not identified using CIViC smMIP sequencing. A subclonal TP53 mutation from AML31 was not identified due to low tumor VAF Originally, this variant was not observed on exome sequencing at $160 \mathrm{X}$, genome sequencing at $314 \mathrm{X}$, or capture sequencing at $1,167 \mathrm{X}$; however, 3 variant supporting reads $(0.04 \%$ VAF) were detected using a capture panel with spike-in probes for recurrently mutated $A M L$ variants $(6,117 X){ }^{22}$ Even though this variant was not identified by the CIViC smMIP sequencing reagents at a sequencing depth of 2,388 , it is likely that increased sequencing depth at this locus would have resulted in variant detection. This is further supported by the fact that other variants with low VAFs (e.g., VAF =1.77\%) were readily identified by the CIViC smMIP reagent. The PTEN variants on SCLC8 and SCLC4 were missed because one or more probes that covered the region of interest showed reproducibly poor performance across samples. Improved balancing of smMIP covering this region, redesign of failed probes, or denser tiling of poor performing regions could improve performance in a subsequent design.

The STK p.T328N variant on CRC5 is noteworthy because it reflects the dynamic nature of academic discovery and illustrates the potential advantage of a panel that permits evolution in response to changing literature. This variant was incidentally found because the original CIViC smMIP capture design was created when STK-LOSS was the only clinically relevant STK variant that 
passed the CIViC actionability score threshold. Given the "LOSS" variant type, the variant only required sparse tiling and approximately 10 MIPs were designed to capture the variant. In July of 2018, Skoulidis et. al. ${ }^{43}$ published an article demonstrating that STK mutations can cause anti-PD-1 resistance. This article was entered into the CIViC database in August of 2018 under STK MUTATION. Curation on this variant raised the CIViC actionability score for STK - MUTATION to >20 points. Therefore, if the reagents had been designed after these findings, STK - MUTATION would have been eligible for CIViC smMIPs design. As such, STK-MUTATION would have been bucketed into the full-tiling cohort, MIPs covering this particular STK variant would have been designed, and the variant would have a CIViC annotation of, "resistance to Pembrolizumab, Nivolumab, and Atezolizumab". This anecdote illustrates the advantages of a dynamic capture panel that can incorporate new discoveries in real-time.

Like all targeted reagents, the preliminary CIViC smMIP design has certain limitations that can be addressed with future iterations. First, the reagent design is limited by the current curation within the CIViC database. Extensive curation from certain groups (e.g., the University Health Network has focused on curating VHL variants) disproportionately increases representation. Conversely, lack of curation in certain areas show a disproportionate decreased representation. Intersection of the CIViC actionability score with existing databases can identify gaps within the database to highlight the need for external and internal curation. Second, targeting additional variants that become eligible as the literature evolves requires designing new probes and rebalancing the reagent. Integrating software to design probes and estimate required probe proportions into $\mathrm{CIViC}$ could help automate this process for future iterations. We also observed in this study that some samples failed quality check due to low sample quality, which potentially limits this approach for degraded samples or samples subjected to formalin-fixed paraffin-embedded (FFPE) preservation, which could potentially be improved with changes to specimen processing or capture procedures. Finally, intersection of variants covered by CIViC smMIPs reagents with clinical relevance summaries from CIViC requires use of the CIViC API, which might be a barrier for some individuals. Future studies will improve visualization of clinical relevance summaries associated with identified variants. Ultimately, after addressing these limitations, we hope to develop, optimize, and validate a clinical CIViC smMIP oncology panel according to the guidelines provided by Jennings et al. ${ }^{44}$ Using the methods provided herein, we plan to build and test panel reagents for a clinical-grade assay validated by current best practices.

In summary, this research validates that community curated data on clinically relevant cancer variants provides an unbiased and dynamic method for capture reagent design. The curated coordinates in the database accurately map to desired variants and probes designed using these coordinates show accurate recapitulation of the genomic landscape described by orthogonal sequencing.

\section{Acknowledgements}

We gratefully acknowledge Tim Ley for sharing genomic data that made this project possible. We also would like to thank the patients and their families for their selfless contribution to the advancement of science. EKB was supported by the National Cancer Institute (T32GM007200 and U01CA209936). Select sample data was funded by the Genomics of AML PPG (T. Ley, PI, P01 CA101937). RU was funded by the National Comprehensive Cancer Network Oncology Research 
Program, general research support provided by Novartis Pharmaceutical Corporation (Novartis), the $\checkmark$ Foundation for Cancer Research, and the National Institute of Dental and Craniofacial Research (NIH NIDCR R01DE024403). RG is funded by the National Cancer Institute (NIH NCl U01CA231844). MG is funded by the National Human Genome Research Institute (NIH NHGRI R0OHG007940). SJS is funded by the National Cancer Institute (NIH NCl R33CA222344). OLG is funded by the $V$ Foundation for Cancer Research, and the National Cancer Institute (NIH NCl U01CA209936 and NIH NCI U01CA231844). This research was supported by a Cancer Moonshot funding opportunity, specifically, an Activities to Promote Technology Research Collaborations (APTRC) for Cancer Research (Admin Supp) award to SJS and OLG under parent awards (R33CA222344 and U01CA209936).

\section{Author Contributions}

EKB completed reagent development, clinical data analysis, wrote code, and wrote the manuscript. $\mathrm{KP}$ conducted sequencing analysis. SJS and AW developed and validated the smMIPs reagents, provided WES data and tumor tissue, and wrote the manuscript. KMC created figures and wrote the manuscript. TAF, RU, and RG, provided WES data and tumor tissue and edited the manuscript. MG, SJS, and OLG supervised the project and revised the paper.

\section{Financial Conflict}

EKB is employed by, owns stock in, and is a board member of Geneoscopy LLC; RG consults for Eli Lilly and Genetech and is a board member/honoraria for EMD Sereno, BMS, Genentech, Pfizer, Nektar, Merck, Celgene, Adaptimmune, GSK, and Phillips Gilmore. 


\section{References and Bibliography}

1. Andrade Nunes R, Nunes RA, Harris LN. The HER2 Extracellular Domain as a Prognostic and Predictive Factor in Breast Cancer. Clin Breast Cancer, 2002, 3:125-35

2. Griffith M, Griffith OL, Smith SM, Ramu A, Callaway MB, Brummett AM, Kiwala MJ, Coffman AC, Regier AA, Oberkfell BJ, Sanderson GE, Mooney TP, Nutter NG, Belter EA, Du F, Long RL, Abbott TE, Ferguson IT, Morton DL, Burnett MM, Weible JV, Peck JB, Dukes A, McMichael JF, Lolofie JT, Derickson BR, Hundal J, Skidmore ZL, Ainscough BJ, Dees ND, Schierding WS, Kandoth C, Kim KH, Lu C, Harris CC, et al. Genome Modeling System: A Knowledge Management Platform for Genomics. PLoS Comput Biol, 2015, 11:e1004274

3. Mardis ER. The 1,000 genome, the 100,000 analysis? Genome Med, 2010, 2:84

4. Collins FS, Varmus H. A New Initiative on Precision Medicine. N Engl J Med, 2015, 372:793-5

5. Gray SW, Hicks-Courant K, Cronin A, Rollins BJ, Weeks JC. Physicians' Attitudes About Multiplex Tumor Genomic Testing. J Clin Oncol, 2014, 32:1317-23

6. Good BM, Ainscough BJ, McMichael JF, Su AI, Griffith OL. Organizing knowledge to enable personalization of medicine in cancer. Genome Biol, 2014, 15:438

7. Dorschner MO, Amendola LM, Turner EH, Robertson PD, Shirts BH, Gallego CJ, Bennett RL, Jones KL, Tokita MJ, Bennett JT, Kim JH, Rosenthal EA, Kim DS, Tabor HK, Bamshad MJ, Motulsky AG, Ronald Scott C, Pritchard CC, Walsh T, Burke W, Raskind WH, Byers P, Hisama FM, Nickerson DA, Jarvik GP. Actionable, Pathogenic Incidental Findings in 1,000 Participants' Exomes. Am J Hum Genet, 2013, 93:631-40

8. Green RC, Berg JS, Grody WW, Kalia SS, Korf BR, Martin CL, McGuire AL, Nussbaum RL, O'Daniel JM, Ormond KE, Rehm HL, Watson MS, Williams MS, Biesecker LG, American College of Medical Genetics and Genomics. ACMG recommendations for reporting of incidental findings in clinical exome and genome sequencing. Genet Med, 2013, 15:565-74

9. Verma A, Rohatgi N, Julka PK, Walia M, Bahl A, Arora RS, Chaturvedi H, Anand AK, Rao R, Gay LM, Kaur J. FoundationOne as a relevant tool for comprehensive genomic profiling and assessment of tumor mutation burden in the era of precision oncology in India. J Clin Orthod, 2017, 35:e23096-e23096

10. Cheng DT, Mitchell TN, Zehir A, Shah RH, Benayed R, Syed A, Chandramohan R, Liu ZY, Won $\mathrm{HH}$, Scott SN, Brannon AR, O'Reilly C, Sadowska J, Casanova J, Yannes A, Hechtman JF, Yao J, Song W, Ross DS, Oultache A, Dogan S, Borsu L, Hameed M, Nafa K, Arcila ME, Ladanyi M, Berger MF. Memorial Sloan Kettering-Integrated Mutation Profiling of Actionable Cancer Targets (MSK-IMPACT): A Hybridization Capture-Based Next-Generation Sequencing Clinical Assay for Solid Tumor Molecular Oncology. J Mol Diagn, 2015, 17:251-64

11. Forbes SA, Beare D, Boutselakis H, Bamford S, Bindal N, Tate J, Cole CG, Ward S, Dawson E, Ponting L, Stefancsik R, Harsha B, Kok CY, Jia M, Jubb H, Sondka Z, Thompson S, De T, Campbell PJ. COSMIC: somatic cancer genetics at high-resolution. Nucleic Acids Res, 2017, 45:D777-83

12. Landrum MJ, Lee JM, Riley GR, Jang W, Rubinstein WS, Church DM, Maglott DR. ClinVar: public archive of relationships among sequence variation and human phenotype. Nucleic Acids Res, 2014, 42:D980-5

13. Gao J, Aksoy BA, Dogrusoz U, Dresdner G, Gross B, Sumer SO, Sun Y, Jacobsen A, Sinha R, Larsson E, Cerami E, Sander C, Schultz N. Integrative analysis of complex cancer genomics and clinical profiles using the cBioPortal. Sci Signal, 2013, 6:I1

14. Cerami E, Gao J, Dogrusoz U, Gross BE, Sumer SO, Aksoy BA, Jacobsen A, Byrne CJ, Heuer ML, Larsson E, Antipin Y, Reva B, Goldberg AP, Sander C, Schultz N. The cBio cancer genomics portal: an open platform for exploring multidimensional cancer genomics data. Cancer 
bioRxiv preprint doi: https://doi.org/10.1101/479394; this version posted December 6, 2018. The copyright holder for this preprint (which was not certified by peer review) is the author/funder, who has granted bioRxiv a license to display the preprint in perpetuity. It is made available under aCC-BY 4.0 International license.

Discov, 2012, 2:401-4

15. Chakravarty D, Gao J, Phillips SM, Kundra R, Zhang H, Wang J, Rudolph JE, Yaeger R, Soumerai T, Nissan MH, Chang MT, Chandarlapaty S, Traina TA, Paik PK, Ho AL, Hantash FM, Grupe A, Baxi SS, Callahan MK, Snyder A, Chi P, Danila D, Gounder M, Harding JJ, Hellmann MD, lyer G, Janjigian Y, Kaley T, Levine DA, Lowery M, Omuro A, Postow MA, Rathkopf D, Shoushtari AN, Shukla N, et al. OncoKB: A Precision Oncology Knowledge Base. JCO Precis Oncol, 2017, 2017

16. Tamborero D, Rubio-Perez C, Deu-Pons J, Schroeder MP, Vivancos A, Rovira A, Tusquets I, Albanell J, Rodon J, Tabernero J, de Torres C, Dienstmann R, Gonzalez-Perez A, Lopez-Bigas N. Cancer Genome Interpreter annotates the biological and clinical relevance of tumor alterations. Genome Med, 2018, 10:25

17. Patterson SE, Liu R, Statz CM, Durkin D, Lakshminarayana A, Mockus SM. The clinical trial landscape in oncology and connectivity of somatic mutational profiles to targeted therapies. Hum Genomics, 2016, 10:4

18. Rubio-Perez C, Deu-Pons J, Tamborero D, Lopez-Bigas N, Gonzalez-Perez A. Rational design of cancer gene panels with OncoPaD. Genome Med, 2016, 8:98

19. Griffith M, Spies NC, Krysiak K, McMichael JF, Coffman AC, Danos AM, Ainscough BJ, Ramirez CA, Rieke DT, Kujan L, Barnell EK, Wagner AH, Skidmore ZL, Wollam A, Liu CJ, Jones MR, Bilski RL, Lesurf R, Feng Y-Y, Shah NM, Bonakdar M, Trani L, Matlock M, Ramu A, Campbell KM, Spies GC, Graubert AP, Gangavarapu K, Eldred JM, Larson DE, Walker JR, Good BM, Wu $\mathrm{C}$, Su Al, Dienstmann R, et al. CIViC is a community knowledgebase for expert crowdsourcing the clinical interpretation of variants in cancer. Nat Genet, 2017, 49:170-4

20. Waalkes A, Penewit K, Wood BL, Wu D, Salipante SJ. Ultrasensitive detection of acute myeloid leukemia minimal residual disease using single molecule molecular inversion probes. Haematologica, 2017, 102:1549-57

21. Eijkelenboom A, Kamping EJ, Kastner-van Raaij AW, Hendriks-Cornelissen SJ, Neveling K, Kuiper RP, Hoischen A, Nelen MR, Ligtenberg MJL, Tops BBJ. Reliable Next-Generation Sequencing of Formalin-Fixed, Paraffin-Embedded Tissue Using Single Molecule Tags. J Mol Diagn, 2016, 18:851-63

22. Griffith M, Miller CA, Griffith OL, Krysiak K, Skidmore ZL, Ramu A, Walker JR, Dang HX, Trani L, Larson DE, Demeter RT, Wendl MC, McMichael JF, Austin RE, Magrini V, McGrath SD, Ly A, Kulkarni S, Cordes MG, Fronick CC, Fulton RS, Maher CA, Ding L, KIco JM, Mardis ER, Ley TJ, Wilson RK. Optimizing cancer genome sequencing and analysis. Cell Syst, 2015, 1:210-23

23. Li H, Durbin R. Fast and accurate short read alignment with Burrows-Wheeler transform. Bioinformatics, 2009, 25:1754-60

24. Li H. Aligning sequence reads, clone sequences and assembly contigs with BWA-MEM. arXiv [q-bioGN], 2013

25. Picard Tools - By Broad Institute, n.d. http://broadinstitute.github.io/picard/. (accessed June 28, 2018)

26. Faust GG, Hall IM. SAMBLASTER: fast duplicate marking and structural variant read extraction. Bioinformatics, 2014, 30:2503-5

27. Larson DE, Harris CC, Chen K, Koboldt DC, Abbott TE, Dooling DJ, Ley TJ, Mardis ER, Wilson RK, Ding L. SomaticSniper: identification of somatic point mutations in whole genome sequencing data. Bioinformatics, 2012, 28:311-7

28. Larson DE, Abbott TE, Wilson RK. Using SomaticSniper to Detect Somatic Single Nucleotide Variants. Curr Protoc Bioinformatics, 2014, 45:15.5.1-8

29. Koboldt DC, Zhang Q, Larson DE, Shen D, McLellan MD, Lin L, Miller CA, Mardis ER, Ding L, Wilson RK. VarScan 2: somatic mutation and copy number alteration discovery in cancer by exome sequencing. Genome Res, 2012, 22:568-76

30. Saunders CT, Wong WSW, Swamy S, Becq J, Murray LJ, Cheetham RK. Strelka: accurate 
bioRxiv preprint doi: https://doi.org/10.1101/479394; this version posted December 6 , 2018. The copyright holder for this preprint (which was not certified by peer review) is the author/funder, who has granted bioRxiv a license to display the preprint in perpetuity. It is made available under aCC-BY 4.0 International license.

somatic small-variant calling from sequenced tumor-normal sample pairs. Bioinformatics, 2012, 28:1811-7

31. McKenna A, Hanna M, Banks E, Sivachenko A, Cibulskis K, Kernytsky A, Garimella K, Altshuler D, Gabriel S, Daly M, DePristo MA. The Genome Analysis Toolkit: a MapReduce framework for analyzing next-generation DNA sequencing data. Genome Res, 2010, 20:1297-303

32. Ye K, Schulz MH, Long Q, Apweiler R, Ning Z. Pindel: a pattern growth approach to detect break points of large deletions and medium sized insertions from paired-end short reads.

Bioinformatics, 2009, 25:2865-71

33. Reble E, Castellani CA, Melka MG, O'Reilly R, Singh SM. VarScan2 analysis of de novo variants in monozygotic twins discordant for schizophrenia. Psychiatr Genet, 2017, 27:62-70

34. Li H, Handsaker B, Wysoker A, Fennell T, Ruan J, Homer N, Marth G, Abecasis G, Durbin R, 1000 Genome Project Data Processing Subgroup. The Sequence Alignment/Map format and SAMtools. Bioinformatics, 2009, 25:2078-9

35. Cibulskis K, Lawrence MS, Carter SL, Sivachenko A, Jaffe D, Sougnez C, Gabriel S, Meyerson M, Lander ES, Getz G. Sensitive detection of somatic point mutations in impure and heterogeneous cancer samples. Nat Biotechnol, 2013, 31:213-9

36. Huang Z. GATK Test Protocol v1 (protocols.io.mhdc326). Protocols.io, 2018. https://doi.org/10.17504/protocols.io.mhdc326

37. Barnell EK, Ronning P, Campbell KM, Krysiak K, Ainscough BJ, Sheta LM, Pema SP, Schmidt AD, Richters M, Cotto KC, Danos AM, Ramirez C, Skidmore ZL, Spies NC, Hundal J, Sediqzad MS, Kunisaki J, Gomez F, Trani L, Matlock M, Wagner AH, Swamidass SJ, Griffith M, Griffith OL. Standard operating procedure for somatic variant refinement of sequencing data with paired tumor and normal samples. Genet Med, 2018. https://doi.org/10.1038/s41436-018-0278-z

38. Hinrichs AS, Karolchik D, Baertsch R, Barber GP, Bejerano G, Clawson H, Diekhans M, Furey TS, Harte RA, Hsu F, Others. The UCSC genome browser database: update 2006. Nucleic Acids Res, 2006, 34:D590-8

39. Pritchard CC, Salipante SJ, Koehler K, Smith C, Scroggins S, Wood B, Wu D, Lee MK, Dintzis S, Adey A, Liu Y, Eaton KD, Martins R, Stricker K, Margolin KA, Hoffman N, Churpek JE, Tait JF, King M-C, Walsh T. Validation and implementation of targeted capture and sequencing for the detection of actionable mutation, copy number variation, and gene rearrangement in clinical cancer specimens. J Mol Diagn, 2014, 16:56-67

40. Lek M, Karczewski KJ, Minikel EV, Samocha KE, Banks E, Fennell T, O'Donnell-Luria AH, Ware JS, Hill AJ, Cummings BB, Tukiainen T, Birnbaum DP, Kosmicki JA, Duncan LE, Estrada K, Zhao F, Zou J, Pierce-Hoffman E, Berghout J, Cooper DN, Deflaux N, DePristo M, Do R, Flannick J, Fromer M, Gauthier L, Goldstein J, Gupta N, Howrigan D, Kiezun A, Kurki MI, Moonshine AL, Natarajan P, Orozco L, Peloso GM, et al. Analysis of protein-coding genetic variation in 60,706 humans. Nature, 2016, 536:285-91

41. Hunter JD. Matplotlib: A 2D Graphics Environment. Comput Sci Eng, 2007, 9:90-5

42. Wagner AH, Devarakonda S, Skidmore ZL, Krysiak K, Ramu A, Trani L, Kunisaki J, Masood A, Waqar SN, Spies NC, Morgensztern D, Waligorski J, Ponce J, Fulton RS, Maggi LB Jr, Weber JD, Watson MA, O'Conor CJ, Ritter JH, Olsen RR, Cheng H, Mukhopadhyay A, Can I, Cessna MH, Oliver TG, Mardis ER, Wilson RK, Griffith M, Griffith OL, Govindan R. Recurrent WNT pathway alterations are frequent in relapsed small cell lung cancer. Nat Commun, 2018, 9:3787

43. Skoulidis F, Goldberg ME, Greenawalt DM, Hellmann MD, Awad MM, Gainor JF, Schrock AB, Hartmaier RJ, Trabucco SE, Gay L, Ali SM, Elvin JA, Singal G, Ross JS, Fabrizio D, Szabo PM, Chang H, Sasson A, Srinivasan S, Kirov S, Szustakowski J, Vitazka P, Edwards R, Bufill JA, Sharma N, Ou S-HI, Peled N, Spigel DR, Rizvi H, Aguilar EJ, Carter BW, Erasmus J, Halpenny DF, Plodkowski AJ, Long NM, et al. STK11/LKB1 Mutations and PD-1 Inhibitor Resistance in KRAS-Mutant Lung Adenocarcinoma. Cancer Discov, 2018, 8:822-35

44. Jennings LJ, Arcila ME, Corless C, Kamel-Reid S, Lubin IM, Pfeifer J, Temple-Smolkin RL, 
bioRxiv preprint doi: https://doi.org/10.1101/479394; this version posted December 6 , 2018. The copyright holder for this preprint (which was not certified by peer review) is the author/funder, who has granted bioRxiv a license to display the preprint in perpetuity. It is made available under aCC-BY 4.0 International license.

Voelkerding KV, Nikiforova MN. Guidelines for validation of next-generation sequencing--based oncology panels: a joint consensus recommendation of the Association for Molecular Pathology and College of American Pathologists. J Mol Diagn, 2017, 19:341-65 


\section{Figures}

Figure 1. Methods for CIViC smMIPs development and validation. The first series describes CIViC smMIPs development. Variants were selected using the sequence ontology ID and the CIViC actionability score. Subsequently, eligible variants were categorized based on length and smMIPs reagents were designed to target regions of interest. The second series describes sample selection and sequencing methods. In total, there were 22 tumor sample derived from 5 tumor subtypes. Of these 27 samples, 15 had tumor and paired normal samples and 7 were tumor-only samples. The third series shows the analysis used to validate CIViC smMIPs design. Variants were called using the pipeline described in the methods, accuracy was attained by comparing original sequencing variants to CIViC smMIPs sequencing variants, and variant allele frequencies across both platforms were also compared.

\section{CIViC smMIPs Reagents}

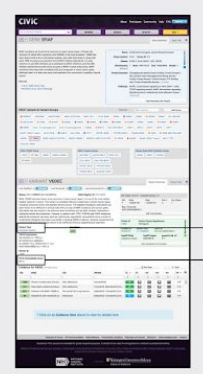

Select variants

Sequence ontology

ID indicates

DNA-based variant

CIViC actionability

score indicates

sufficient curation
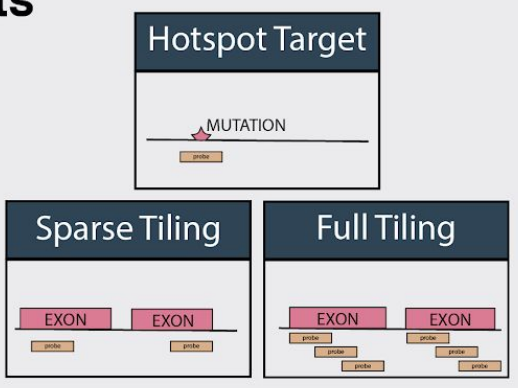

Categorize variants

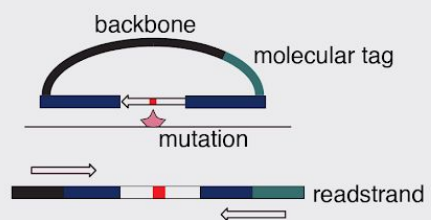

pooled
readstrands

Develop MIPs

\section{Validation samples}

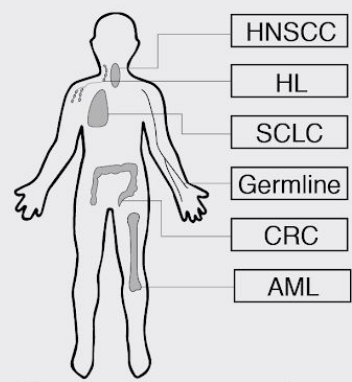

Select samples

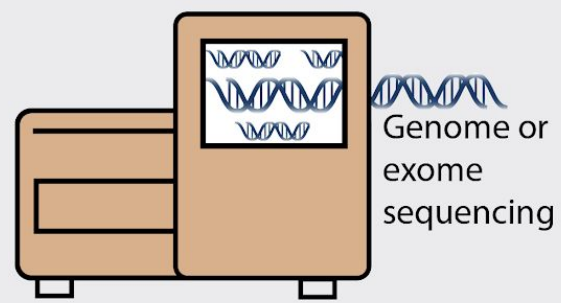

Original sequencing

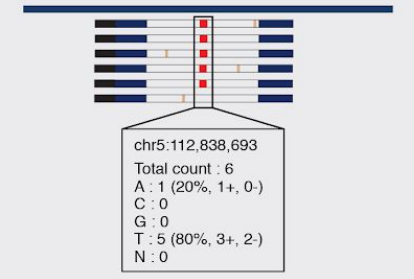

smMIPs sequencing

\section{Platform comparison}

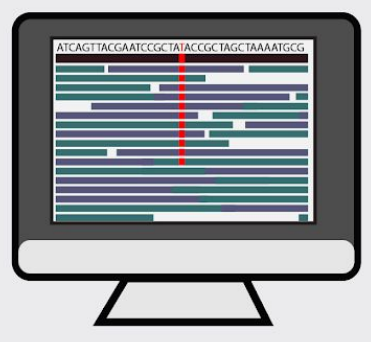

Call variants

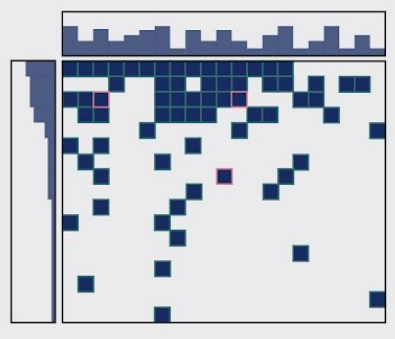

Compare variants

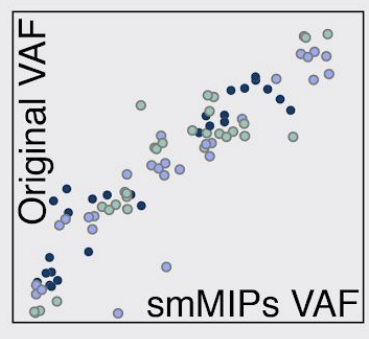

Compare VAFs 
Figure 2. Regions targeted by the CIViC single molecule molecular inversion probes (smMIPs) are, by design, supported by extensive clinical relevance according to the CIViC database. Variants that were eligible for CIViC smMIPs development were bucketed into various coverage methods based on sequence ontology identification number (SOID) and length. Bar graph shows total number of evidence items used for each of the buckets parsed by the evidence type.

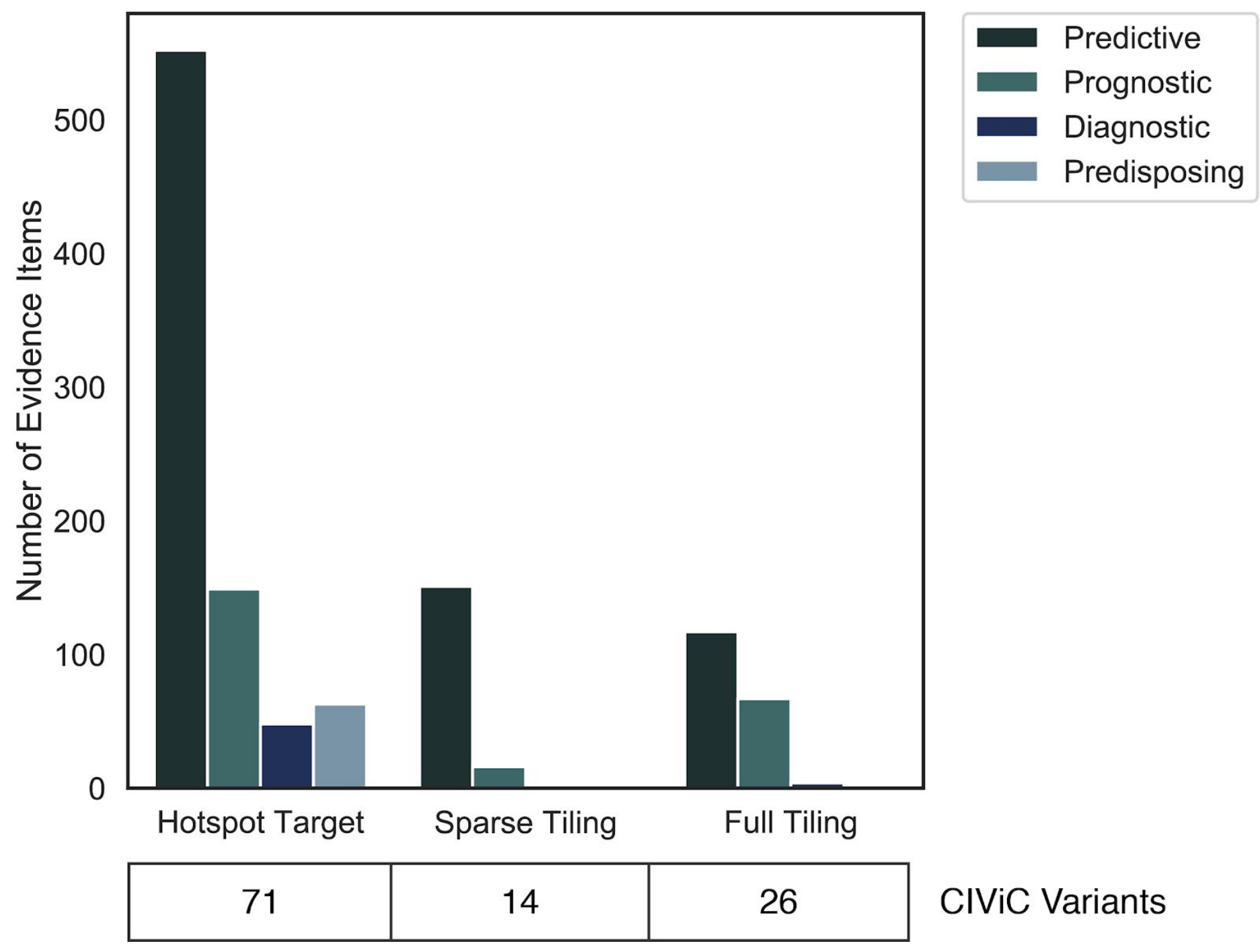


Figure 3. Waterfall plot shows extensive overlap between variants observed using original exome or genome sequencing with variants observed using CIViC smMIPs sequencing. Each column represents a sample that had original exome or genome sequencing with subsequent orthogonal validation using the CIViC smMIPs sequencing. Rows represent mutated genes across all samples. Numbers within each box represent the variant allele frequency (VAF) observed on original exome or genome sequencing. Green boxes indicate that a variant was observed by CIViC smMIPs and validated with original exome or genome sequencing. Tan boxes indicate the the variant was observed on original exome or genome sequencing but not identified via the CIViC smMIPs sequencing. The left panel indicates the number of samples containing a mutation in the indicated gene.

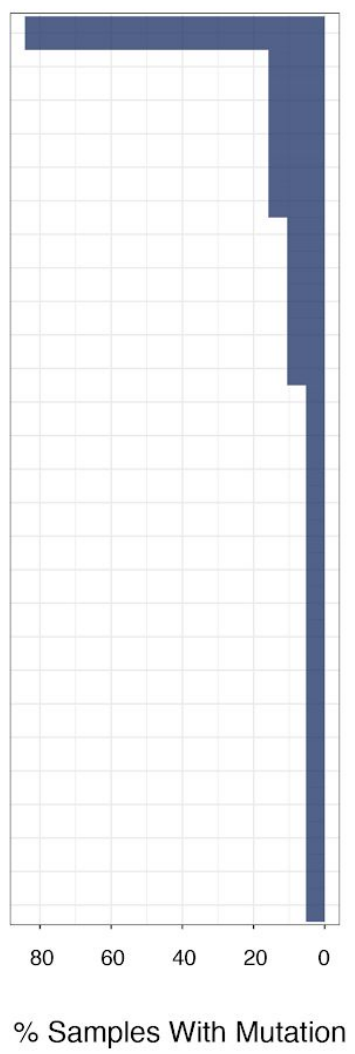

Waterfall plot for 70 variants observed on original sequencing

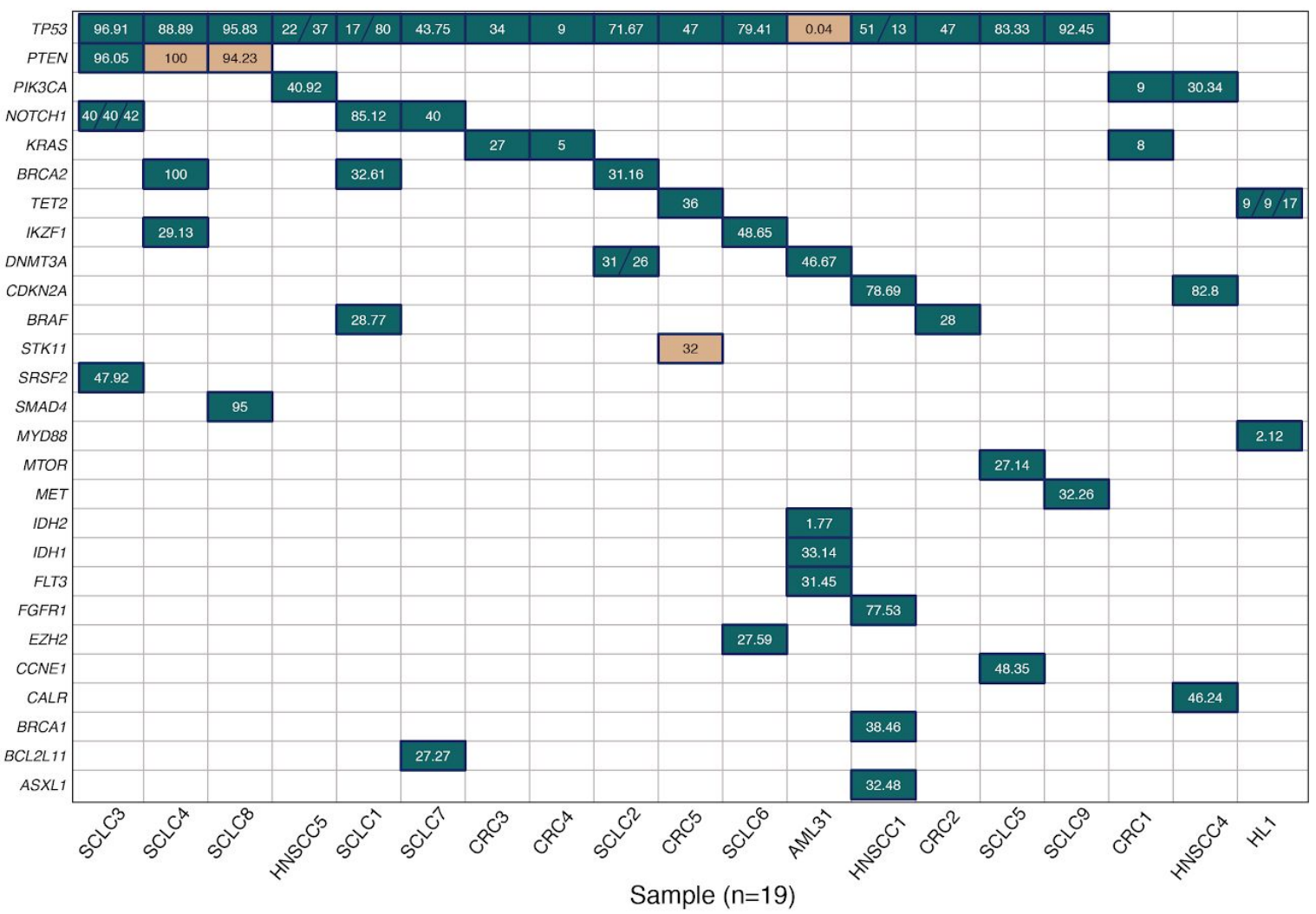

Legend for box color and value

Observed on original sequencing and not validated on smMIPs sequencing

Observed on original sequencing and validated on smMIPs sequencing

\# Original sequencing VAF 
Figure 4. Variant allele frequencies (VAFs) observed using original exome or genome sequencing correlates with VAFs observed using CIViC smMIPs sequencing a Correlation of VAF with original sequencing parse by sequencing status (i.e., passed sequencing if total sequencing counts $>1$ standard deviation from mean and tag complexity $>600,000)$. b Correlation of VAF with validation status (e.g., 'True' if the variant identified using exome/genome sequencing was identified on CIViC smMIPs sequencing). $\mathbf{c}$ Correlation of VAF parsed by tumor type. $\mathbf{d}$ Correlation of VAF parsed by DNA mass input for library construction. e Correlation of VAF parsed by depth of sequence coverage. $\mathbf{f}$ Correlation of VAF parsed by presence or absence of matched normal tissue. $\mathbf{g}$ Correlation of VAF parsed by sample identity.

a

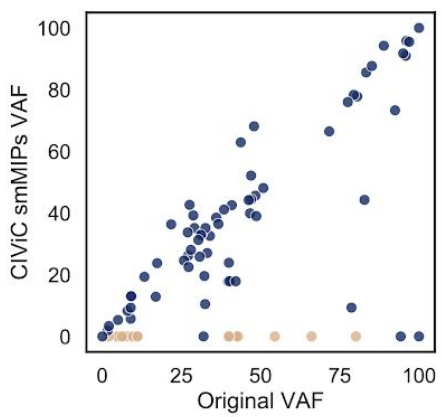

b

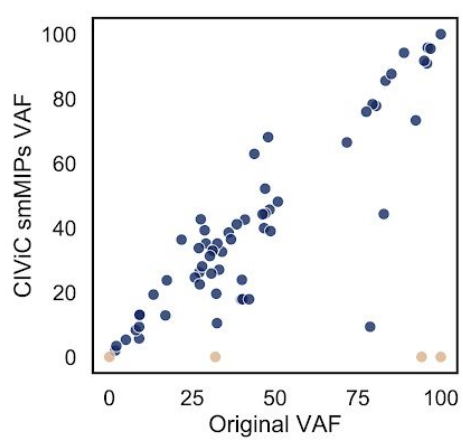

C

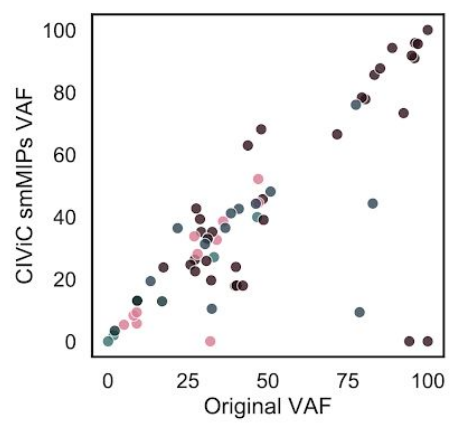

d

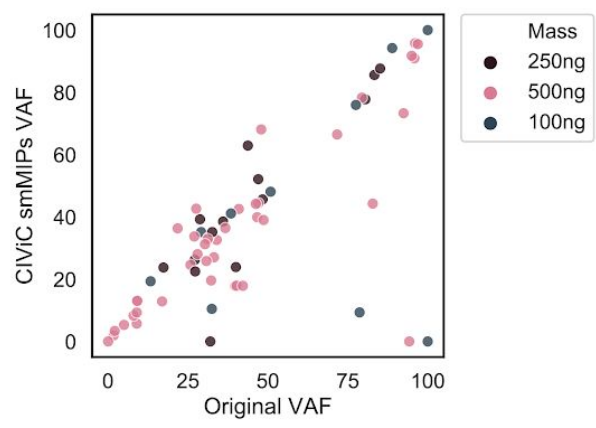

e

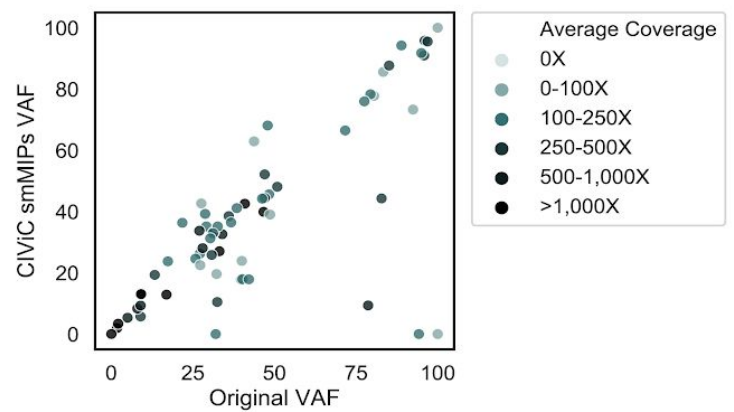

f

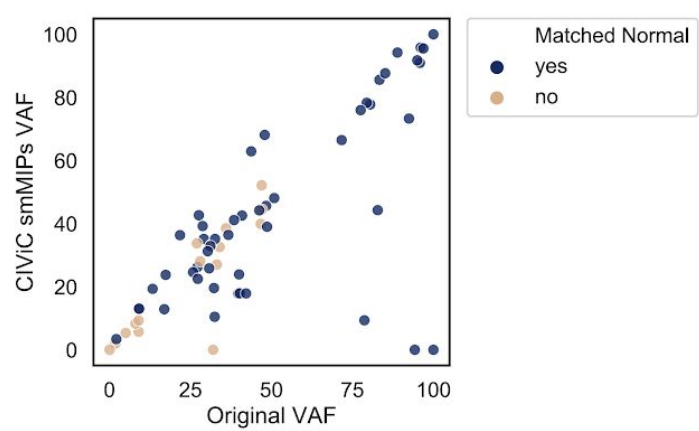

g

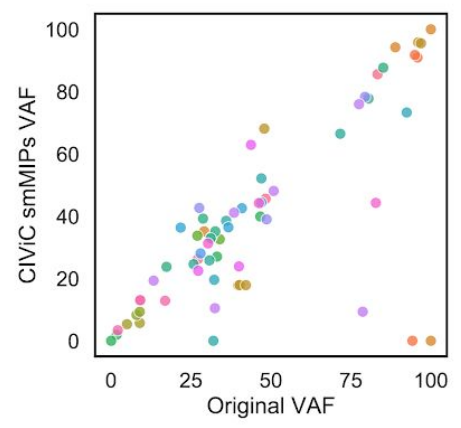

sample
SCLC_5
SCLC_8
SCLC_4
SCLC_3
CRC_4
CRC_1
CRC_3
AML_31
SCLC_1
SCLC_2
CRC_5
SCLC_9
OSCC_5
CRC_2
SCLC_6
OSCC_1
SCLC_7
OSCC_4
HL_1 
Figure 5. Analysis of variants rescued by CIViC smMIPs sequencing for samples with both tumor and matched normal. There were 217 variants called as somatic by the CIViC smMIPs sequencing that were not identified on original sequencing. All variants were manually reviewed using both CIViC smMIPs sequencing data and original sequencing data a. During manual review 55 variants were identified as germline. A histogram shows that the distribution of the smMIPs VAF for these germline variants are observed at $50 \%$ and $100 \%$ VAF, indicating heterozygosity and homozygosity, respectively. b. An additional 36 variants were identified as sequencing artifacts. Most artifacts were either mononucleotide repeats (MN), dinucleotide repeats (DN), or tandem repeats (TR). Other artifacts include multiple mismatches (MM) or multiple variants (MV). c. During manual review, 162 variants did not show any support in the original sequencing data. Most unsupported variants did not have sufficient coverage to be detected based on a binomial probability of at least 4 variant-supporting reads (see Methods). d. The remaining 11 variants had variant support in original sequencing but were not called as somatic in final original annotation. Scatter plot shows correlation between original VAF and CIViC smMIPs VAF for these variants.

a

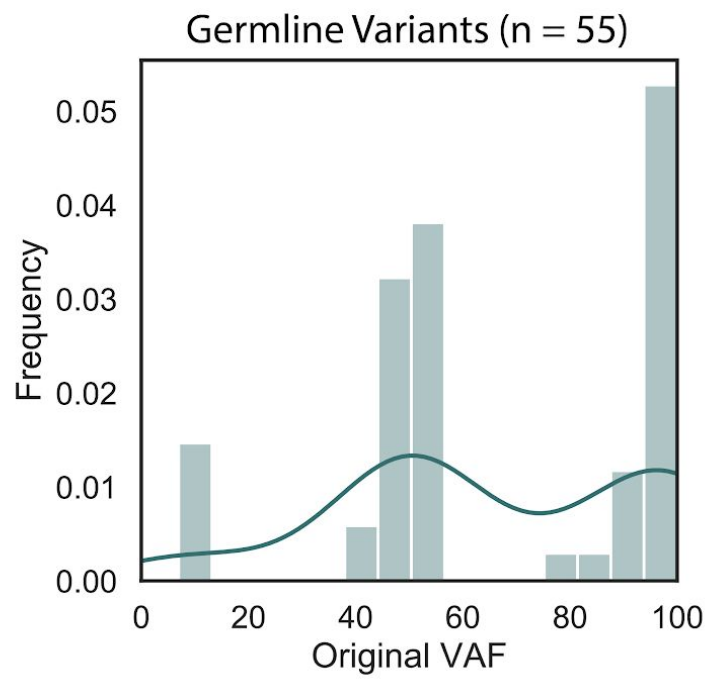

b

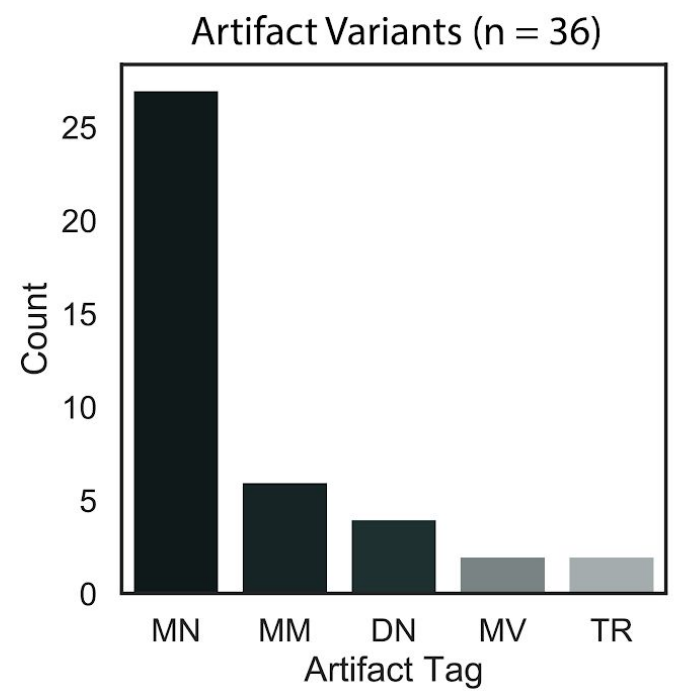

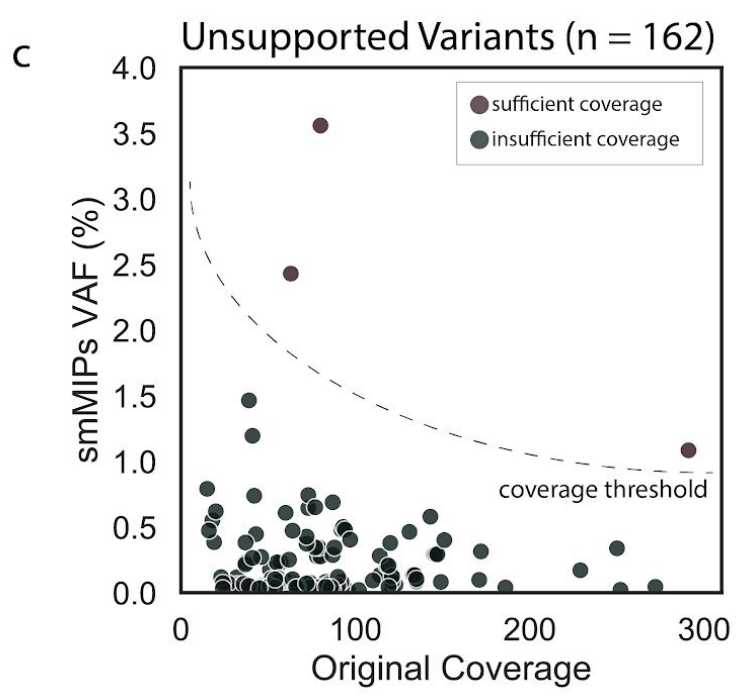

d

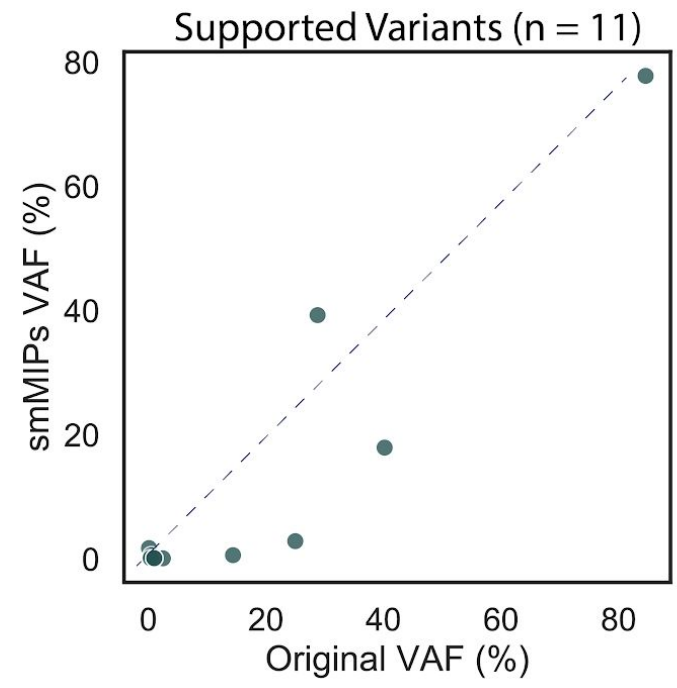




\section{Supplementary Tables and Figures}

Supplementary Tables:

https://docs.google.com/spreadsheets/d/1iYc-EYtNNMd9eSqeKZqKU0TQMdmP6aZJ4ZS3oF3BW5s/ edit?usp=sharing

Supplementary Table 1. List of existing pan-cancer capture reagents with description on genes and development.

Supplementary Table 2. Sequence ontology identification numbers (SOIDs) with sequencing category.

Supplementary Table 3. Variants and associated genes eligible for CIViC smMIPs design after filtering based on CIViC actionability score and SOID.

Supplementary Table 4. Sequencing data availability for samples used in analysis.

Supplementary Table 5. Samples used to validate the CIViC smMIPs reagents with variants and variant allele frequencies identified using whole exome sequencing.

Supplementary Table 6. The CIViC database was used to elucidate clinical relevance for the 218 variants missed by original sequencing. For each of the 12 samples that had matched tumor/normal pairs, the clinical relevance statement, associated evidence item ID, and the pubmed ID are listed. 


\section{Supplementary Figures}

\section{Supplementary Figure 1. Example of CIViC interface for BRAF V600E mutation with}

highlighted features. The clinical relevance summary provides an overview of the clinical relevance of the gene. Individual variants and variant groups associated with the gene are subsequently listed for selection. Selecting a variant (i.e., V600E) allows the user to see the clinical relevance summary for the variant and additional curated information (i.e., sequence ontology label, variant coordinates, clinical assertion, and $\mathrm{CIViC}$ actionability score, etc.). Evidence statements supporting the variant can be viewed by clicking on the rows in the evidence statement grid. This provides the user with additional information on how the clinical relevance statements and actionability score were generated.

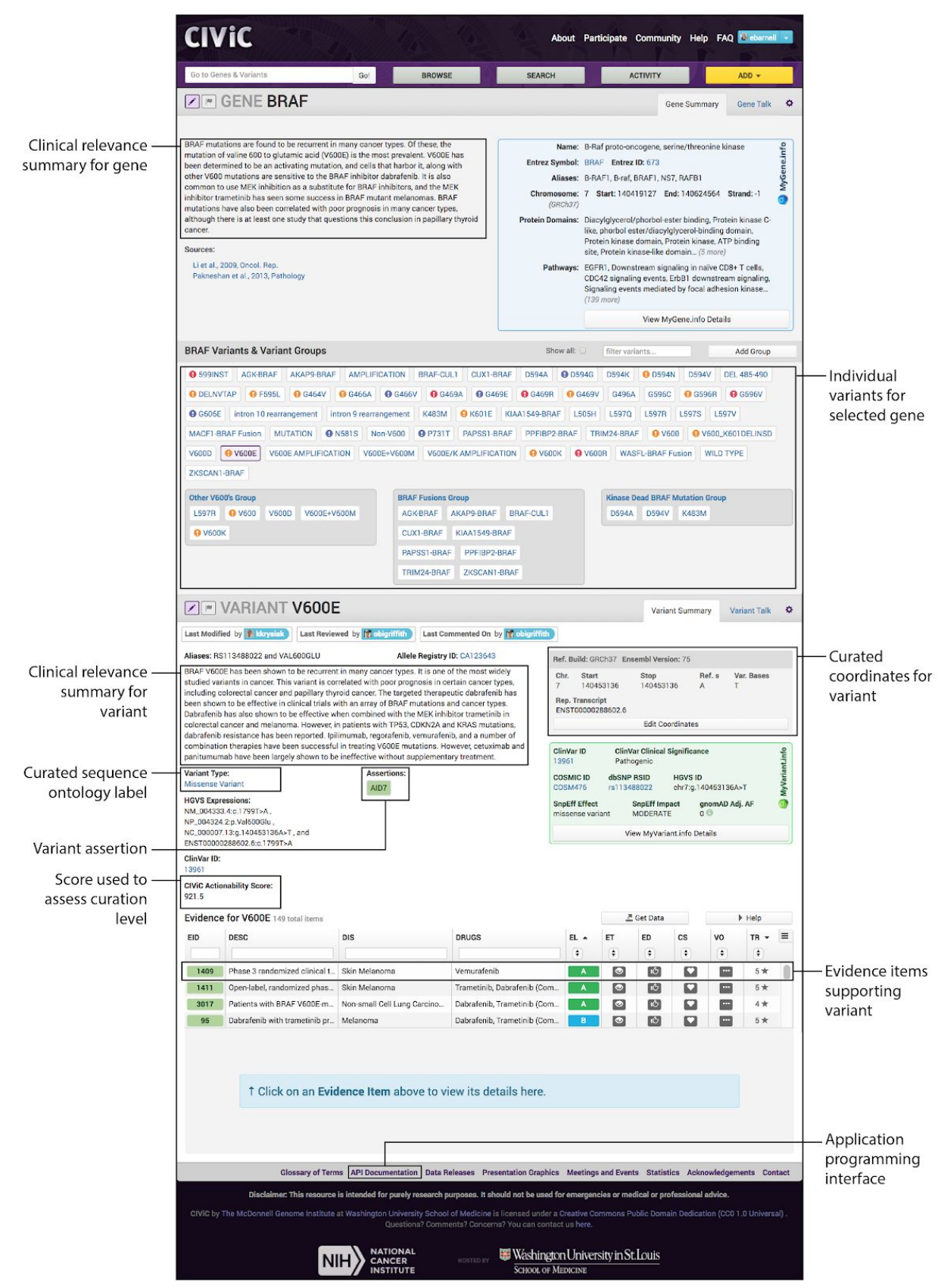


bioRxiv preprint doi: https://doi.org/10.1101/479394; this version posted December 6, 2018. The copyright holder for this preprint (which was not certified by peer review) is the author/funder, who has granted bioRxiv a license to display the preprint in perpetuity. It is made available under aCC-BY 4.0 International license.

\section{Supplementary Figure 2. The CIViC actionability score provides relative level of curation for variants} within the CIViC database. To calculate the actionability score, first evidence item scores are calculated by multiplying the associated evidence level point by the trust rating point. For each variant, evidence item scores are summed and the CIViC actionability score is generated.

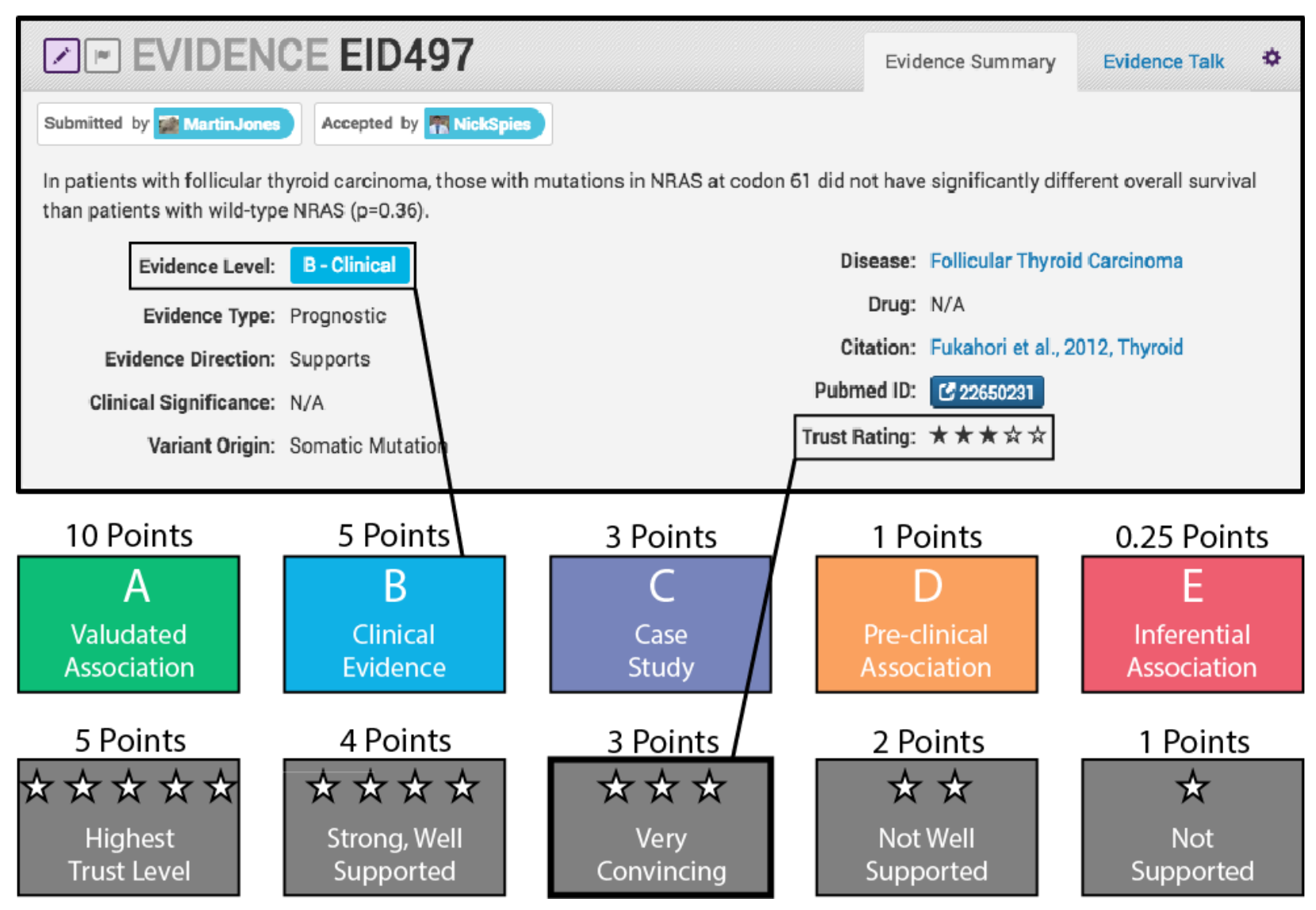

